\title{
Synthesis and Characterization of an Oxo-Centered Homotrimetallic Uranium(IV)- Cyclobutadienyl Dianion Complex
}

Josef T. Boronski, Laurence R. Doyle, Ashley J. Wooles, John A. Seed, Stephen T. Liddle*

Department of Chemistry, The University of Manchester, Oxford Road, Manchester, M13 9PL, UK.

*Correspondence email: steve.liddle@manchester.ac.uk

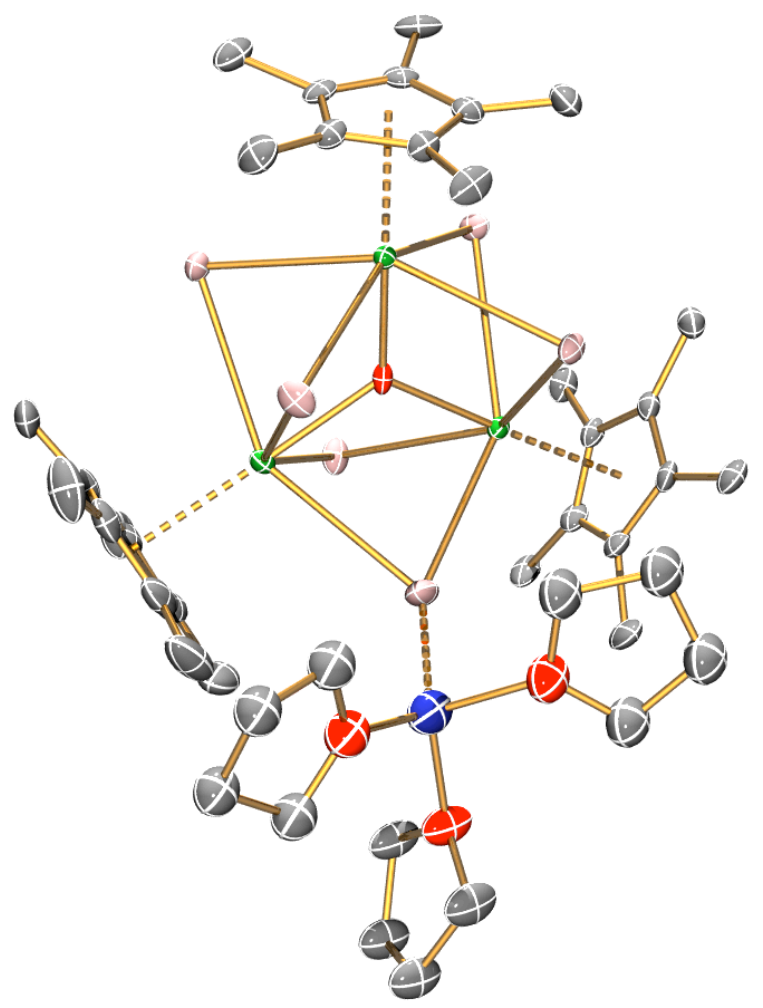

Figure S1. The $\left[\left\{\mathrm{U}\left(\mu^{2}-\mathrm{I}\right)_{2} \mathrm{Cp} \mathrm{p}^{*}\right\}_{3}\left\{\mu^{3}-\mathrm{O}\right\}\left\{\mathrm{Li}(\mathrm{THF})_{3}\right\}\right]$ fragment of the solid state structure of 3 . Displacement ellipsoids are at $30 \%$. Disordered components, $\left[\mathrm{Li}(\mathrm{THF})_{4}\right]^{+}$counter cation and hydrogen atoms are omitted for clarity. 


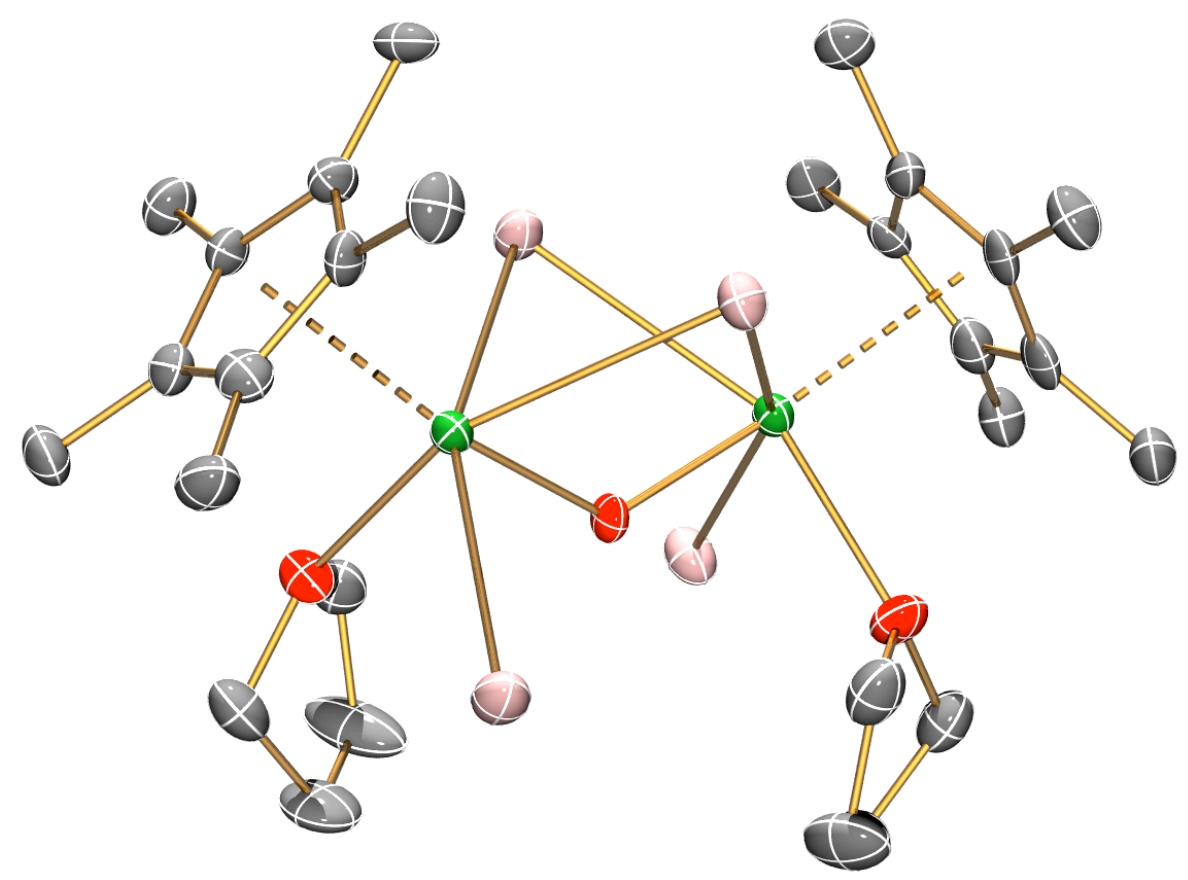

Figure S2. The solid state structure of $\left[\left\{\mathrm{U}\left(\eta_{5}-\mathrm{C}_{5} \mathrm{Me}_{5}\right)(\mu-\mathrm{I})(\mathrm{I})(\mathrm{THF})\right\}_{2}(\mu-\mathrm{O})\right]$. Displacement ellipsoids are at 50\%. Hydrogen atoms are omitted for clarity.

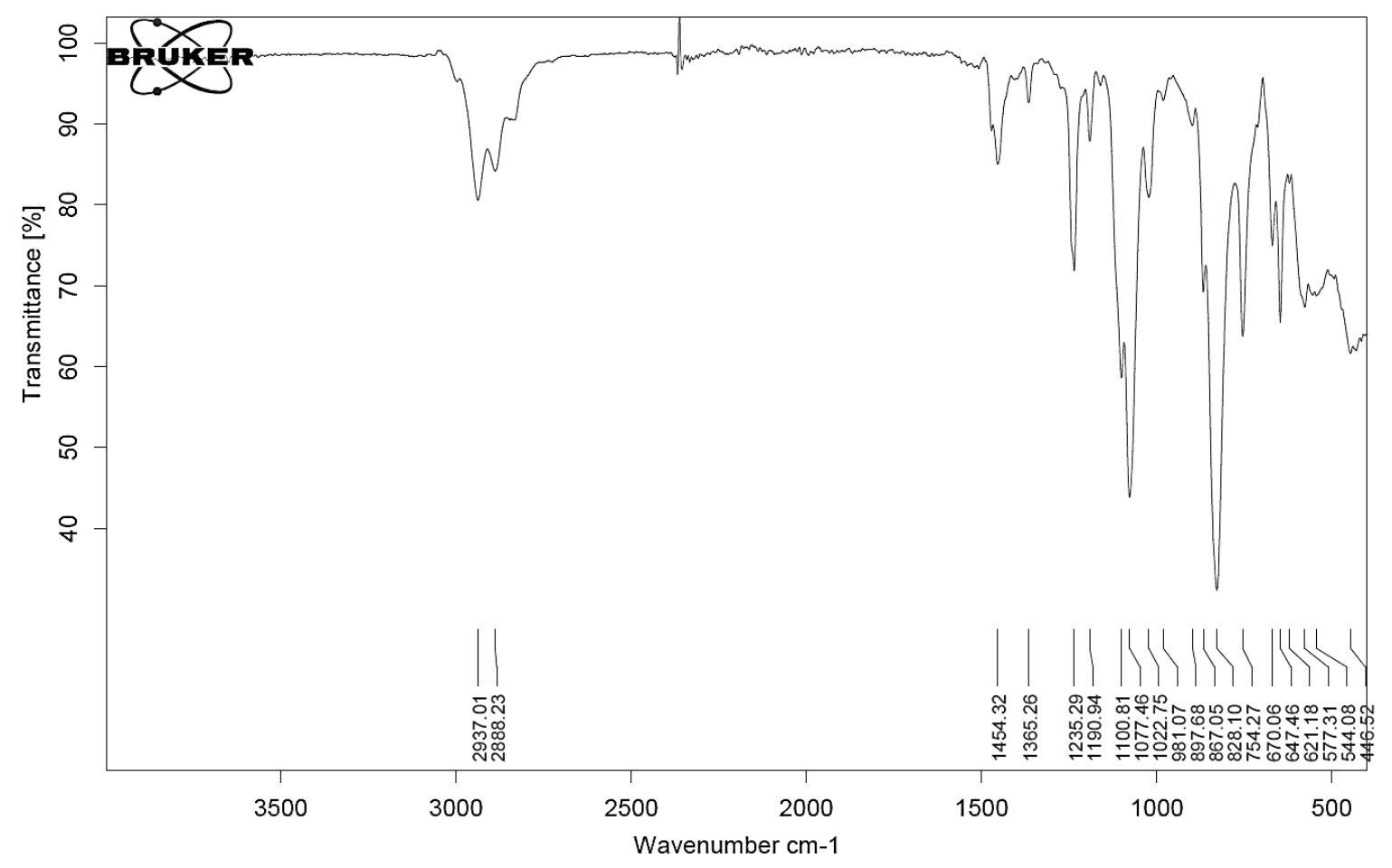

Figure S3. FTIR spectrum of 5. 

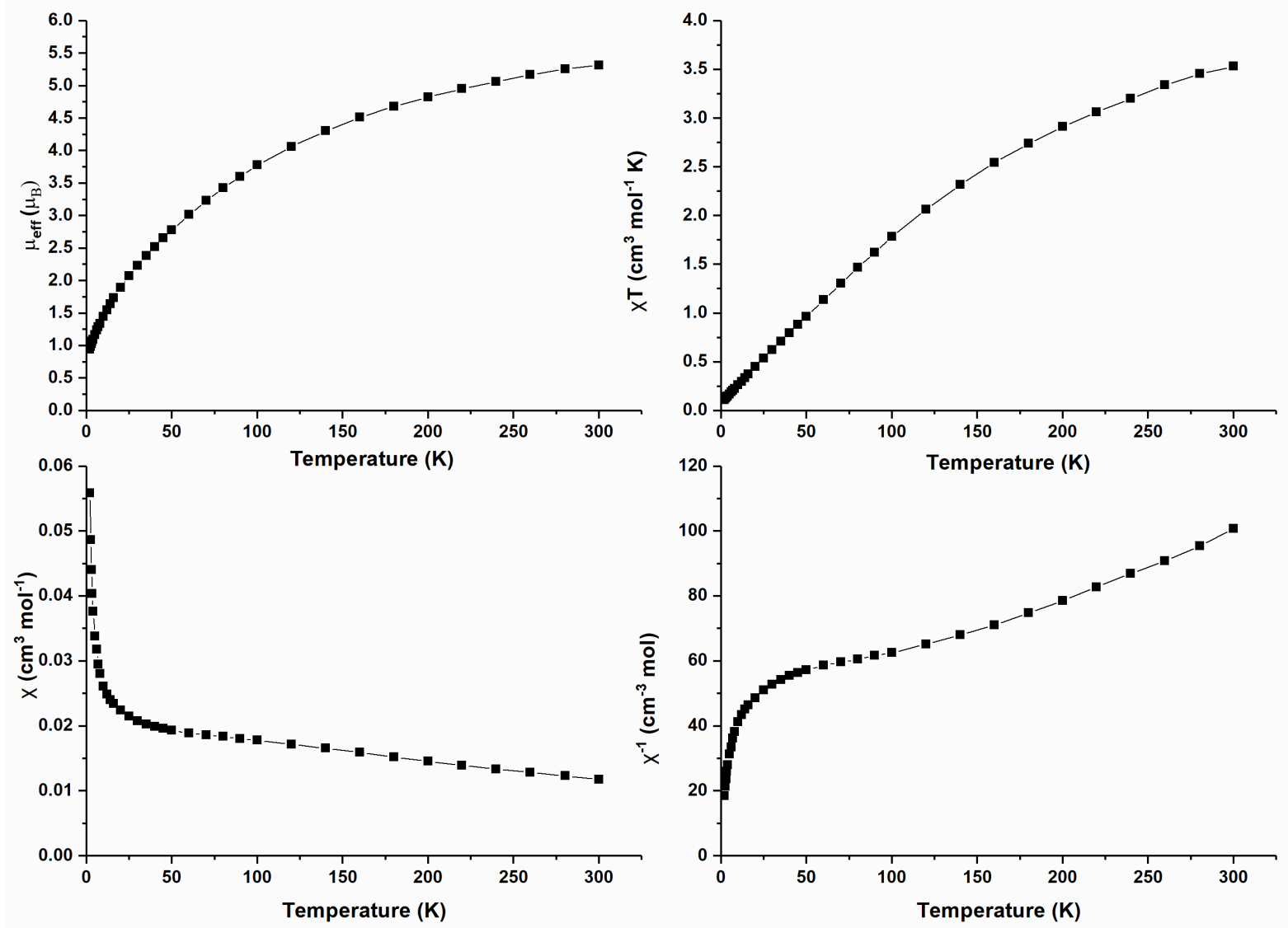

Figure S4. Variable temperature SQUID magnetometry plot for 5 over the temperature range 2$300 \mathrm{~K}$. The line is a guide to the eye only. 
Table S1. Crystallographic Details

\begin{tabular}{|c|c|c|c|}
\hline Compound & 3 & 5 & $\begin{array}{l}{\left[\left\{\mathrm{U}\left(\mathrm{C}_{5}-\mathrm{C}_{5} \mathrm{Me}_{5}\right)(\mu-\right.\right.} \\
\left.\mathrm{I})(\mathrm{I})(\mathrm{THF})\}_{2}(\mu-\mathrm{O})\right]\end{array}$ \\
\hline Formula & $\mathrm{C}_{88} \mathrm{H}_{146} \mathrm{I}_{12} \mathrm{Li}_{2} \mathrm{O}_{9} \mathrm{U}_{6}$ & $\mathrm{C}_{102} \mathrm{H}_{156} \mathrm{D}_{30} \mathrm{I}_{6} \mathrm{MgO}_{7} \mathrm{Si}_{12} \mathrm{U}_{3}$ & $\mathrm{C}_{28} \mathrm{H}_{46} \mathrm{I}_{4} \mathrm{O}_{3} \mathrm{U}_{2}$ \\
\hline Fw, $\mathrm{g} \mathrm{mol}^{-1}$ & 4312.90 & 3391.56 & 1414.31 \\
\hline Cryst size, $\mathrm{mm}$ & $0.141 \times 0.108 \times 0.043$ & $0.099 \times 0.057 \times 0.055$ & $\begin{array}{c}0.222 \times 0.092 \times \\
0.065\end{array}$ \\
\hline Crystal system & monoclinic & monoclinic & triclinic \\
\hline Space group & $\mathrm{C} 2 / \mathrm{c}$ & $\mathrm{C} 2 / \mathrm{c}$ & P-1 \\
\hline Temperature (K) & $150(2)$ & $100(2)$ & $150(2)$ \\
\hline $\mathbf{a},(\AA)$ & $22.4507(5)$ & $22.9173(7)$ & $8.44100(10)$ \\
\hline b, $(\AA)$ & $12.5672(2)$ & $24.0524(6)$ & $12.1233(2)$ \\
\hline c, $(\AA)$ & $43.0931(8)$ & $23.8826(7)$ & $18.6362(2)$ \\
\hline$\alpha,\left(^{\circ}\right)$ & 90 & 90 & $76.3180(10)$ \\
\hline$\beta,\left(^{\circ}\right)$ & $96.217(2)$ & $93.706(3)$ & $83.7070(10)$ \\
\hline$\gamma,\left(^{\circ}\right)$ & 90 & 90 & $73.0500(10)$ \\
\hline $\mathrm{V},\left(\AA^{3}\right)$ & $12086.9(4)$ & $13136.9(6)$ & $1770.79(4)$ \\
\hline $\mathbf{Z}$ & 4 & 4 & 2 \\
\hline$\rho_{\text {calc }} \mathrm{g} \mathrm{cm}^{-3}$ & 2.370 & 1.715 & 2.653 \\
\hline$\mu, \mathrm{mm}^{-1}$ & 46.700 & 5.258 & 53.113 \\
\hline Reflections measured & 29705 & 46658 & 18757 \\
\hline $\begin{array}{c}\text { No. of unique reflections, } \\
\text { Rint }\end{array}$ & $12098,0.0564$ & $16320,0.0378$ & $7008,0.0582$ \\
\hline $\begin{array}{l}\text { No. of reflections with } F^{2} \\
\qquad>2 s\left(F^{2}\right)\end{array}$ & 10968 & 12687 & 6839 \\
\hline $\begin{array}{c}\text { Transmission coefficient } \\
\text { range }\end{array}$ & $0.587-0.856$ & $0.740-0.835$ & $0.013-0.238$ \\
\hline$R, R_{\mathrm{w}}{ }^{a}\left(F^{2}>2 \mathrm{~s}\left(F^{2}\right)\right)$ & $0.0571,0.1520$ & $0.0462,0.1059$ & $0.0527,0.1407$ \\
\hline$R, R_{\mathrm{w}}{ }^{a}$ (all data) & $0.0627,0.1553$ & $0.0681,0.1137$ & $0.0536,0.1419$ \\
\hline$S^{\mathrm{a}}$ & 1.035 & 1.026 & 1.107 \\
\hline Parameters, Restraints & 615,555 & 552,49 & 344,0 \\
\hline $\begin{array}{l}\text { Max.,min. difference } \\
\text { map, e } \AA^{-3}\end{array}$ & $2.845,-3.407$ & $4.490,-1.628$ & $3.297,-4.665$ \\
\hline
\end{tabular}

